\title{
$\checkmark$ Research Square \\ Effects of a Snack on Performance and Errors During a Simulated 16-h Night Shift: a Pilot Study
}

Sanae Oriyama ( $D$ oriyama@hiroshima-u.ac.jp)

Hiroshima University

Kotomi Yamashita

Hiroshima University

\section{Research Article}

Keywords: nighttime eating, night shift, nurse, performance, sleepiness

Posted Date: March 22nd, 2021

DOI: https://doi.org/10.21203/rs.3.rs-334882/v1

License: (c) (1) This work is licensed under a Creative Commons Attribution 4.0 International License. Read Full License 


\section{Abstract}

Background: Night shift workers might not eat due to their busy schedules during the night shift. However, diet may not only satisfy hunger, but also affect performance and errors.

The aim of this study was to clarify the effect of a snack on performance and errors during 2-day, 16-h simulated night shifts.

Methods: A randomized repeated-measure crossover study was performed to investigate subjective and cognitive performance in 15 healthy female adults (mean age, 21.7 years) after they consumed a snack (352 kcal) during a simulated night shift (16:00 to 09:00). The participants were kept awake from wake up in the morning to the next day at 09:00. Subjects were tested for performance on the Uchida-Kraepelin test, as well as for subjective feeling, body temperature, psychomotor vigilance test, and heart rate variability, before and after they consumed the snack. One day before the experiment, all participants wore an actigraphy monitoring device to determine their sleep state.

Results: There was no difference between the snack condition and the skipping condition in sleep states the day before the experiment. On the day of the experiment, between 16:00 and 09:00, subjective sleepiness, fatigue, and body temperature were not different between the two conditions. Subjects showed a significant improvement in performance on the Uchida-Kraepelin test and total errors on the psychomotor vigilance test, the primary outcome measure, when they consumed a snack compared with the skipping condition. In addition, the snack condition was associated with decreased high-frequency power, decreased low-frequency power/high-frequency power ratio, and increased heart rate, in the vagally mediated heart rate variability indices, which may reflect a higher ability to modulate cognitive and behavioral processes.

Conclusions: These results suggest that providing a snack to shift workers during night shifts might improve work safety and efficiency.

Trial registration: This study was registered with the University Hospital Medical Information NetworkClinical Trials Registry (UMIN-CRT registry ID: UMIN 000034345).

\section{Background}

As Japan is increasingly becoming a 24-h society [1], rotating shifts have come to incorporate nighttime work, which has led to the necessity of shift work in a variety of service sectors. However, compared with the day shift, shift work can pose threats to the health of workers and increase the risk of developing various health problems. Despite these risks, the numbers of shift workers in Japan has continued to rise [1]. The healthcare professions in Japan, especially nurses, represent a typical example of an industry that has increasingly adopted a shift work system. Since 2017, the number of nurses working 16-h night shifts in Japan has increased significantly [2], which has resulted in increased physical and mental burdens. Nurses working on such schedules often experience morning sleepiness and fatigue $[3,4]$. In 
addition, it has been reported that long working hours are associated with a higher risk of errors and accidents and poorer performance [5]. Compared with the traditional 8-h working day, the risk of occupational injury when working more than $12 \mathrm{~h}$ per day is estimated to be $147 \%$ higher [6].

Night work has been reported to increase morning sleepiness because of disruptions in circadian rhythms [7]. In addition, regarding dietary patterns during night shifts, avoiding eating altogether has been investigated as a strategy to maintain alertness [8]. A previous study [9] investigating the effects of food intake during nighttime (from 01:30 to 02:30) found that fasting resulted in reduced performance in a cognitive vigilance task. Another study reported that eating a snack during the night shift (at 01:30) alleviated hunger without leading to perceived fullness or increased sleepiness until 05:00 [10], although the snack's effect duration has remained unknown. Portion size is an important aspect of nighttime eating. Among nurses, reduced meal sizes have been reported as a strategy for minimizing gastric symptoms during night shifts [8]. However, to the best of our knowledge, the duration of the effects of food intake and the potential impact on performance have not been reported in previous studies investigating the effects of sleep loss on performance. Given this background and the fact that performance is most severely impaired at night, reducing food intake during night shifts may serve as a suitable countermeasure. If the duration of the snack effect could be clarified, it could help answer the core questions of both what and when night shift workers should eat to avoid metabolic disturbances; this, in turn, could optimize wakefulness and performance and help prevent accidents in the workplace. Accordingly, in this study, a rice ball was selected as a midnight snack that night shifters can easily eat even if they are busy. Rice is a staple food for more than half of the world's population. In addition to calories, rice is a good source of magnesium, phosphorus, manganese, selenium, iron, folic acid, thiamin, and niacin, but it is low in fiber and fat.

Therefore, this study aimed to investigate the impact of eating (the Snack condition) compared with not eating (the Skipping condition) at night on performance, sleepiness, fatigue, and other physiological measures during 16-h simulated night shifts. We hypothesized that under the conditions of extended wakefulness during the "biological night," eating a snack would lead to decreased errors and maintain performance until the morning. To test this hypothesis, sleepiness, hunger, vigilant attention, cognitive processing, and other physiological measures were assessed under both Snack and Skipping conditions during consecutive 2-day, 16-h simulated shifts throughout the night.

\section{Methods}

\section{Participants}

The participants in this randomized, crossover study were 15 healthy adult females (mean age \pm standard deviation [SD], $21.7 \pm 0.5$ years; body mass index, $19.81 \pm 2.45 \mathrm{~kg} / \mathrm{m}^{2}$ ) recruited between August and September 2018. None of the subjects had any previous night shift experience, and none was identified as morning type or evening type according to the morningness-eveningness questionnaire [11]. All of the participants were current nonsmokers, in the luteal phase of their menstrual cycle [12,13], and 
had normal sleep patterns (habitual sleep ranging between 7 and $9 \mathrm{~h}$ ). No participants were currently taking any prescribed medications. The required sample size was determined to be 14 (actual power $81.0 \%$ ) based on an effect size, a error, and power (1-b) of $0.25,0.05$, and 0.8 , respectively. The power calculation in this study was carried out using G*Power 3 [14].

Study meal

All participants consumed one or two meals between 16:00 and 09:00. Under both the Snack and Skipping conditions, all participants ate a meal (containing $708 \mathrm{kcal}, 19.4 \mathrm{~g}$ protein, $17.9 \mathrm{~g}$ fat, $112.4 \mathrm{~g}$ carbohydrates, and $3.5 \mathrm{~g}$ sodium) at 19:30 (not eating at night), and under the Snack condition only, participants ate a snack at 03:30 (eating at night). The meal provided at 03:30 consisted of two rice balls (containing $352 \mathrm{kcal}, 5.8 \mathrm{~g}$ protein, $1.8 \mathrm{~g}$ fat, $75.8 \mathrm{~g}$ carbohydrates, and $1 \mathrm{~g}$ sodium) and two slices of yellow pickled radish. Many night shift workers prefer a high-energy diet rich in carbohydrates [15], such as the rice balls that are widely available at any convenience store in Japan. All participants were given $20 \mathrm{~min}$ to finish their meals, and they were encouraged to eat everything on their plate.

Study protocol

A flowchart of this study is shown in Figure 1. The measurements were conducted over 2 consecutive days between 16:00 and 09:00. Each experiment day had 5 participants who were randomly assigned to one of two conditions using counterbalancing. All participants were instructed to refrain from strenuous physical exercise and not to consume caffeine or alcohol for $24 \mathrm{~h}$ prior to and during each study day. Two days before the experiment began, all participants wore an actigraphy monitoring device (ActiGraph; Ambulatory Monitoring Inc., Ardsley, NY, USA) on their non-dominant wrist and recorded their sleep and activity levels in a diary.

On the day of the experiment, all participants arrived at the laboratory at 15:00. Until 16:00, they carried out practice assessments, including the Uchida-Kraepelin test (UKT) and the psychomotor vigilance test (PVT). The Snack condition involved consuming a meal and a snack at 19:30 and 03:30, whereas the Skipping condition involved consuming a meal at 19:30 only. At the start of each experiment, the participants were fitted with a heart rate variability (HRV) sensor (GMS Inc., Tokyo, Japan). For each hour throughout the experiment, they were given $10 \mathrm{~min}$ to record their sublingual temperature once and complete the visual analog scale for sleepiness, fatigue, and hunger, 10 min to perform the UKT, and 10 min to measure the PVT. The next 20 min were considered free time, and the remaining 10 min were considered a rest period. The participants spent their free time reading, drawing, or drinking water. During the 10-min rest period, they sat on chairs and chatted with the other participants. The same meal amounts and contents were given to the participants between 19:30 and 19:50 in each experimental period. The HRV sensor was removed at the end of each experiment, but the participants were asked to continue wearing the actigraphy monitoring device until they woke up the next day. During all waking times, the participants remained awake in the laboratory and were continuously monitored by the researchers. 
All participants stayed in a windowless and sound-insulated laboratory for 2 consecutive days ( 1 night) (Figure 1). The laboratory environment was maintained at $26 \pm 2{ }^{\circ} \mathrm{C}[16]$ and $50 \%$ relative humidity under indoor illumination on the table at $200 \mathrm{~lx}$.

Measurements

Sleep parameters

The sleep parameters measured were total sleep time, sleep efficiency (total sleep time / time in bed ' 100), sleep onset latency, and wake after sleep onset. All parameters were measured using the actigraphy monitoring device, and they were analyzed using the AW2 software package (Ambulatory Monitoring Inc.).

Physiological parameters

Sublingual temperature

The circadian rhythm of body temperature is one of the most frequently used indicators of circadian rhythmicity [17], and body temperature has been shown to be related to sleepiness, fatigue, and performance of a single-digit mental arithmetic task [18]. Sublingual temperature, which is considered an index of internal body temperature [19], was measured hourly using an oral thermometer (MC-612; Omron Inc., Kyoto, Japan) to assess changes in circadian modulation during the night.

Cognitive performance

Subjective assessment of sleepiness, fatigue, and hunger

A visual analog scale was used for the subjective assessment of sleepiness, fatigue, and hunger [20]. The participants rated their sleepiness, fatigue, and hunger every hour on a 100-mm line, with values ranging from $0 \mathrm{~mm}$ (not sleepy, tired, or hungry at all) to $100 \mathrm{~mm}$ (extremely sleepy, tired, or hungry).

Uchida-Kraepelin Test (UKT)

The UKT (Nisseiken, Tokyo, Japan), a serial mental arithmetic task, was used to measure cognitive performance. This test is a questionnaire that requires intense concentration and effort, and it has been used as a tool to induce mental stress [21]. The test material consisted of a pre-printed paper with 20 rows of 115 random, single-digit figures. The subjects' task was to add adjacent figures horizontally, and then write the one-digit answer between the 2 figures; they were asked to proceed along each row as quickly and as accurately as they could in a 1-min period. On being given the first cue, the subjects began calculating from the first row. Then, when the second cue was given after $1 \mathrm{~min}$, the subjects were required to begin a new row, without regard to their position on the current row. This procedure was repeated 8 more times, for the total duration of $10 \mathrm{~min}$. The sum of the correct answers for each 1-min period over the 10-min task was used as the value for the analysis. 
The PVT is a reaction time task considered to be a sensitive measure for assessing the effects of sleep loss [22]. In this study, a precise computer-based version of the 10-min PVT was used to avoid problems of uncertainty with regard to the accuracy of the test platform timing [23]. All participants were instructed to look at a computer monitor and press a response button when a white circular edge appeared on the screen; pressing the response button stopped the counter and displayed the response time (in milliseconds) for a 1-s period. The PVT measures response times to visual stimuli randomly occurring at 2- to 10-s intervals over a 10-min period [23]. The outcome measures for the PVT include the median response time, number of lapses (response time $>500 \mathrm{~ms}$ ), and total errors (incorrect responses), as well as mean response time.

Autonomic nervous system activity

For the purposes of the present study, HRV was obtained through autoregressive analysis of $\mathrm{R}-\mathrm{R}$ intervals measured between 16:00 and 09:00. All data were analyzed offline after analog-to-digital conversion of $250-\mathrm{Hz} \mathrm{R}-\mathrm{R}$ waves. HRV was measured every 5 min during each hour and then averaged; these measurements were used to monitor autonomic nervous system activity throughout the night [24]. High-frequency (HF) and the low-frequency/high-frequency (LF/HF) are used as indicators of cardiac parasympathetic and cardiac sympathetic nervous activity, respectively $[25,26]$. The LF/HF power ratio indicates the balance between sympathetic and parasympathetic outflows [27].

Statistical analysis

All results are shown as mean \pm SD or standard error of the mean. All sleep variables measured the day before the experiment were analyzed using the $t$-test.

To test the effects of consuming a snack on neurobehavioral and physiological outcomes during the early morning measurement periods, a fully saturated, linear mixed-effects analysis of variance was carried out [28], with a between-participant fixed effect of condition and a within-participant fixed effect of time (at 03:00 vs. from 04:00 to 09:00) and a random intercept. Within-condition comparisons were used to minimize the effect of individual differences. Multiple comparisons were assessed using the Bonferroni correction to evaluate patterns of change under the two conditions. As a secondary analysis, between-condition hourly comparisons from 16:00 to 09:00 were analyzed using the Mann-Whitney $U$ test.

To assess the postprandial effect of the meal throughout the testing time, the net incremental area under the curve (niAUC), calculated from pre- (at 03:00) and postprandial time points, was tested. All statistical analyses were conducted using SPSS (version 22.0J; IBM, Tokyo, Japan). The hypothesis rejection level for all tests was set at $p<.05$, and a notable trend was set at $p<.1$.

Ethical considerations 
This study was approved by the Center for Integrated Medical Research of Hiroshima University (study protocol ID No.: C-252). Written, informed consent was obtained from all participants before the first examination. The study protocol conformed to the Declaration of Helsinki guidelines. This study was registered with the University Hospital Medical Information Network-Clinical Trials Registry (UMIN-CRT registry ID: UMIN 000034345) after the enrollment of participants had begun. The authors confirm that all ongoing and related trials for this intervention have been registered.

\section{Results}

Sleep state before the experiment

Total sleep time, sleep efficiency, sleep onset latency, go to bed time, and wake up time, assessed by the ActiGraph, were not different between phases (Table 1).

Table 1

Sleep sensitivity parameters by meal condition

\begin{tabular}{|llll|}
\hline Variable & Snack & \multicolumn{1}{l}{ Skipping } & p $₫$ value \\
\hline ActiGraph & & & \\
\hline Total sleep time, min & $461.9(65.1)$ & $470.5(73.4)$ & .735 \\
\hline Sleep efficiency, \% & $93.6(5.4)$ & $94.5(6.0)$ & .663 \\
\hline Sleep onset latency, min & $10.9(33.3)$ & $7.5(16.7)$ & .732 \\
\hline Go to bed time, h:min & $00: 30(01: 21)$ & $23: 54(01: 07)$ & .562 \\
\hline Wake up time, h:min & $08: 26(01: 21)$ & $07: 57(01: 04)$ & .288 \\
\hline Date are means (SD), N=15 & & \\
\hline
\end{tabular}

Physiological parameters

Outcomes regarding the participants' physiological parameters, cognitive performance, and autonomic nervous system activity from 16:00 to 09:00 are shown in Figures 2 and 3. From 16:00 to 03:00, no significant main effect of their interaction was observed, and the analysis from 03:00 to 09:00 is shown in Table 2. 
Table 2

Linear mixed models: physiological parameters, cognitive performance, and autonomic nervous system activity outcome measures

\begin{tabular}{|c|c|c|c|c|c|c|}
\hline \multirow[t]{2}{*}{ Outcome Measure } & \multicolumn{2}{|l|}{ Condition } & \multicolumn{2}{|l|}{ Time } & \multicolumn{2}{|c|}{ Condition*time } \\
\hline & $\mathrm{Fdf}$ & $p$ & $\mathrm{~F} \mathrm{df}$ & $p$ & $\mathrm{~F} \mathrm{df}$ & $p$ \\
\hline \multicolumn{7}{|l|}{ Physiological parameter } \\
\hline Temperature & $1.690_{1,182}$ & .195 & $1.2976,182$ & .260 & $1.004_{6,182}$ & .424 \\
\hline \multicolumn{7}{|l|}{ Cognitive performance } \\
\hline Subjective sleepiness & $.166_{1,182}$ & .684 & $6.246_{6,182}$ & $<.001$ & $.688_{6,182}$ & 659 \\
\hline Fatigue & $.5921,182$ & .443 & $11.4696,182$ & $<.001$ & $.3326,182$ & .919 \\
\hline Hunger & $135.1981,182$ & $<.001$ & $12.7636,182$ & $<.001$ & $5.8986,182$ & $<.001$ \\
\hline Kraepelin performance & $16.124_{1,182}$ & $<.001$ & $10.9506,182$ & $<.001$ & $.5366,182$ & .780 \\
\hline PVT (Mean RT) & $.005_{1,182}$ & .942 & $6.5916,182$ & $<.001$ & $.1546,182$ & .988 \\
\hline PVT (Median RT) & $.3721,182$ & .542 & $5.288_{6,182}$ & $<.001$ & $.344_{6,182}$ & .913 \\
\hline PVT 囚Lapse】 & $.116_{1,182}$ & .734 & $6.764_{6,182}$ & $<.001$ & $.576_{6,182}$ & .749 \\
\hline PVT $₫$ Total errors $\rrbracket$ & $5.150_{1,182}$ & .025 & $4.3236,182$ & $<.001$ & $1.335_{6,182}$ & .244 \\
\hline \multicolumn{7}{|l|}{ Autonomic nervous } \\
\hline $\mathrm{HR}$ & $32.036_{1,169}$ & $<.001$ & $1.816_{1,169}$ & .099 & $2.411_{6,169}$ & .029 \\
\hline $\mathrm{LF} / \mathrm{HF}$ & $1.045_{1,169}$ & .308 & $3.0686,169$ & .007 & $1.914_{6,169}$ & .081 \\
\hline HF & $11.930_{1,169}$ & .001 & $1.6676,169$ & .132 & $1.016_{6,169}$ & .417 \\
\hline \multicolumn{7}{|c|}{$\begin{array}{l}\text { Results shown are from linear mixed model analyses with main effects of condition: Snack } \\
\text { condition/Skipping condition; time from 03:00 to 09:00 (interval between } 1 \mathrm{~h} \text { ) and their interactions } \\
\text { (condition*time). PVT: Psychomotor Vigilance Test; HR: heart rate; LF/HF: low-frequency power/high- } \\
\text { frequency power ratio; HF: high-frequency power, bold indicates a significant value }(\mathrm{p}<.05) \text {, F-static: } \\
\text { variation between sample means, fd: degrees of freedom. }\end{array}$} \\
\hline
\end{tabular}

Cognitive performance

The interaction between condition and time of measure was not significant for sleepiness and fatigue, such that sleepiness and fatigue increased in the two conditions from 03:00 to 09:00; a similar trend was seen for across the night to early morning (Figure 2B, C, left panel). 
The interaction between eating condition and time of measure was significant for hunger $(p<.001$; Table 2). As can be seen in Figure 2D (left panel), hunger decreased in the Snack condition compared to the Skipping condition $(p<.001)$ from 04:00 to 09:00.

The results of the UKT are shown in Figure 3A. Significant main effects of condition were observed for the number of correct answers, in which the number of correct answers performed from 04:00 to 09:00 was significantly better in the Snack condition than in the Skipping condition $(p<.001)$.

PVT

Results regarding the mean RT, lapse, and total errors on the PVT from 03:00 to 09:00 are shown in Figure 3B-D. On PVT, mean RT, and lapse, there were no significant main or interaction effects. On the other hand, total errors showed a significant main effect of condition $(p=.025)$; total errors were significantly fewer under the Snack condition than under the Skipping condition (Figure 3D).

Autonomic nervous system activity

Electrocardiogram data could not be obtained from one participant during the experiment; therefore, data were analyzed for 14 participants. Figure 4A shows the heart rate data. Significant main effects of condition $(p<.001)$ and their interaction were observed $(p=.029)$. Under the Snack condition, heart rate increased compared with the Skipping condition at 04:00 $(p=.028), 05: 00(p=.046), 06: 00(p=.028)$, and 07:00 $(p=.078)$.

A significant main effect of condition was observed for high-frequency power (Figure 4B), which tended to be significantly lower under the snack than under the Skipping condition between 04:00 and 09:00 ( $p=$ .001). In addition, there was a significant main effect of time $(p=.007)$, and a marginally significant condition * time interaction ( $p=.081)$ was seen for the low-frequency /high-frequency (Figure 4C). In the Snack condition, the low-frequency /high-frequency was significantly lower at 03:00 ( $p=.041), 04: 00(p=$ $.038), 06: 00(p<.001), 07: 00(p<.001)$, and 08:00 $(p<.001)$ after eating than at 02:00 before eating.

\section{Discussion}

The present study investigated the effects of nighttime eating during night shifts on temperature, subjective sleepiness, fatigue, hunger, vigilant attention, processing speed, and autonomic nervous system activity during testing between 16:00 and 09:00. Increasing perceptions of hunger across night shifts have been reported by shift workers, who have described hunger as a factor that influences their decision to eat during the night shift [8]. In the present study, consuming a snack (352 kcal) during the night reduced perceptions of hunger, maintained better performance, and increased heart rate for $3 \mathrm{~h}$. To the best of our knowledge, this study was the first to investigate each hour the effects of consuming a snack on objective nighttime sleepiness and fatigue, vigilant attention, information processing, and changes in autonomic nervous system activity over a simulated night shift in a controlled laboratory environment. 
Regardless of the Snack condition, the greatest impairments in subjective sleepiness and fatigue, vigilant attention, and performance increased toward morning. This could explain the increases in circadian sleep pressure and decreased alertness throughout the night [29]. Consistent with our previous study [30], although sleepiness and fatigue increased across the simulated 16-h night shift, no differences were found between participants that had and had not eaten during the night $[10,31]$. Whereas the results after 04:00 showed increased subjective sleepiness and fatigue [32], this might correspond with the circadian low points between 02:00 and 06:00 [29]. Previous studies have suggested that snacking (10\% 24-h estimated energy requirement) at 00:30 did not increase sleepiness at 02:30 and 05:00 [10], but eating at 01:30 is not a suitable mealtime, because sleepiness increased at 03:00. In the present study, sleepiness and fatigue did not differ, regardless of eating. It is speculated that rice balls are mainly carbohydrates, low in fat and relatively slow to digest and absorb, and their granular shape is presumed to require chewing; in addition to the effect on motor control, chewing increases the arousal level and alertness [33]. Thus, these factors may have prevented a significant increase in drowsiness [33]. Therefore, we concluded that the ingestion of the snack (352 kcal) did not change the subjects' sleepiness and fatigue. Performance on the PVT reflects circadian modulations in neurobehavioral functions, in addition to the effects of sleep pressure that develop with an increased duration of waking time, without being confounded by a learning curve [34]. PVT, mean RT, and lapse were similar between the two conditions. Subjective sleepiness is more likely to be underestimated [35] than objectively evaluated sleepiness [35]. Drowsiness weakens alertness and is likely to cause human error. In this study, the snack at 03:30 was affected by circadian modulations in neurobehavioral functions that developed in association with waking time and sleep pressure; decreases were seen in attention, but the PVT total errors were significantly higher in the Skipping condition than in the Snack condition. In particular, night shift workers are prone to errors at dawn, but it has been shown that light meals may make it possible to prevent accidents in the morning.

According to recent reviews, breakfast consumption may result in acute improvements of memory, attention, and motor and executive function, although no conclusion about the effect of macronutrients on cognitive function has been reached [33]. The present result shows that a small snack lessened the decline in the number of correct answers on the UKT. The UKT, which is used to measure cognitive task performance, involves simple mental arithmetic and handwriting [33]. Several cognitive domains, including sustained attention and short-term memory, are involved in the mental arithmetic task. Handwriting is also a complex perceptual-motor skill [35]. Given recent studies highlighting the potential metabolic consequences of consuming a large nighttime meal [36,37], a small meal, such as two rice balls, may be an option that is more readily available to maintain the cognitive function of shift workers.

These findings indicate that the subjects who ate a small meal felt less hungry for $5 \mathrm{~h}$ (i.e., until 09:00), and that those who did not eat at night perceived greater hunger toward morning. Although these findings may predominantly reflect a longer inter-meal interval, hunger is known to display a circadian rhythm and be increased during the night. Restricted sleep also increases hunger and appetite, and results in a preference for a high-energy diet rich in carbohydrates [38]. Night work has been reported to cause mental stress [39], which can trigger fatigue and lead to decreased performance [40,41]. As short-term changes in 
food intake are known to affect various aspects of cognitive function, we believe that reducing the stress induced by hunger may help sustain performance. Compared with high-fat meals, those rich in carbohydrates reduce mental (in contrast to physical) performance and increase sleepiness [42]. Eating a heavy lunch is associated with significant increases in motor vehicle accidents and a tendency to experience greater subjective sleepiness [43]. More generally, objective signs of sleepiness typically peak about $3.5 \mathrm{~h}$ after eating [44]. In other words, in this respect, tasks that require sustained attention are the most sensitive, with larger meals producing more frequent lapses of attention [45]. However, in the present study, the consumption of two rice balls (containing approximately $352 \mathrm{kcal}$ of primarily carbohydrates) did not appear to affect sleepiness and fatigue.

In the present study, the heart rate was significantly higher under the snack than under the Skipping condition from 04:00 to 06:00. When eating a meal, visceral circulating blood volume increases to support the process of digestion and absorption, the sympathetic nervous system supports it, and the heart rate increases [46]. The contribution of increased sympathetic nervous system activity postprandially to the thermic effect of food is not always evident, and it has been shown to depend on the size and composition of the meal, with the clearest effect seen with carbohydrates. The brain integrates signals related to food intake from various sites (e.g., gut, hepatoportal area, chemoreceptors), leading to increased peripheral sympathetic outflow [46]. Few studies have focused on the relationship between meals and autonomic nervous system activity. The results of the present study showed that a small meal increased the heart rate and decreased parasympathetic nervous system activity, which is related to rest and digestive activity. The two systems, the sympathetic nervous system and the parasympathetic nervous system, generally act in a complementary fashion, because the increase in one is usually associated with a decrease in the other. Many sympathetic nervous system functions are opposite to those of the parasympathetic nervous system. In the present results for the low-frequency power/high-frequency power ratio values between 02:00 and 09:00, the Skipping condition showed no change, but the Snack condition resulted in an immediate decrease after eating. Because the lowfrequency power/high-frequency power ratio has been shown to represent changes in sympathetic nervous activity in the autonomic nervous system as a stress index [47], stress levels may have been lower under the eating condition. Consuming a meal is reportedly effective for reducing stress [48], which is consistent with the results of the present study. Among night shift nurses who remain awake for a long period of time, sleepiness and fatigue increase, and stress levels also gradually rise with increased workloads around the end of their shifts [49]. If stressful situations are prolonged, night shift workers may develop chronic fatigue. The results of this study suggest that eating a small meal at 03:30 may be effective for reducing stress in the morning. In addition, shift workers often report that increased alertness is one of the main factors that influences their decision to eat during a shift [10]. Dietary strategies and altered eating behaviors have been reported as strategies to maintain alertness during the night shift among a sample of nurses [50]. Furthermore, reduced meal sizes have been reported as a strategy used by nurses to minimize gastric symptoms during night shifts. Therefore, promoting the consumption of a small meal at 03:30 during break time may help night shift workers maintain their health and improve safety during 16-h night shifts, as well as prevent malpractice and chronic fatigue. 
In the case of long night shifts, the time for taking of meals is restricted, and it is difficult to eat freely. We recommend a small snack to maintain performance and stop the increase in errors regardless of time. Rice balls, which can be easily eaten even when busy, may be one of the options for meals during night shifts.

\section{Limitations}

This study has several limitations. First, it was conducted under laboratory conditions; therefore, the degree of change in performance and sleepiness could vary from that under actual working conditions, which greatly depend on differences in workload due to the nature of the work or the timing of busy work periods. To confirm effective measures for reducing sleepiness and fatigue tailored to the conditions of specific professions and workplaces, intervention studies need to be conducted in actual workplaces. It is possible that the present results would be different if actual shift workers had been studied; therefore, caution is needed when interpreting the results. Second, the participants in this study were all young women in their 20s with no shift work experience, which may have affected the results. We would like to investigate this issue further in a future study.

A previous study reported that taking a nap during a night shift helped nurses recover from night shift fatigue [51]. Therefore, in the future, it will be necessary to take measures to reduce drowsiness and fatigue by combining meals and naps.

\section{Conclusions}

This study investigated the effects of eating a meal at night during night shifts on subjective sleepiness, vigilant attention, processing speed, hunger, and autonomic nervous system activity between 16:00 and 09:00. The results showed that consuming a small meal (352 kcal) at 03:30 during the night shift reduced hunger and helped sustain performance until morning. To the best of our knowledge, this is the first study to explore the effects of consuming a small meal at night on 16-h performance in a controlled laboratory environment.

\section{Abbreviations}

PVT: Psychomotor vigilance test

HRV: Heart rate variability

UKT: Uchida-Kraepelin Test

SD: Standard deviation

HR: Heart rate

LF/HF: Low-frequency /High-frequency 
HF: High-frequency

\section{Declarations}

\section{Availability of data and materials}

The datasets used and analyzed during the current study are available from the corresponding author on reasonable request.

\section{Acknowledgements}

Not applicable.

\section{CONSORT}

This study adheres to consort guidelines.

\section{Funding}

This research was funded by Japan Society for the Promotion of Science, KAKENHI Grant Number JP26293452. The Japan Society for the Promotion of Science had no role in the design of the study, the collection, analysis or interpretation of data, or the writing of this paper.

\section{Authors' contributions}

SO contributed to the conception and design, analysis, interpretation of data, and manuscript drafting. KY contributed to interpretation of data. All authors reviewed and commented on subsequent drafts of the manuscript.

\section{Ethics declarations}

\section{Ethics approval and consent to participate}

This study was approved by the Center for Integrated Medical Research of Hiroshima University (study protocol ID No.: C-252). Written, informed consent was obtained from all participants before the first examination. The study protocol conformed to the Declaration of Helsinki guidelines. This study was registered with the University Hospital Medical Information Network-Clinical Trials Registry (UMIN-CRT registry ID: UMIN 000034345, full date registration 1/10/2018) after the enrollment of participants had begun. The authors confirm that all ongoing and related trials for this intervention have been registered.

\section{Consent for publication}

Not applicable.

\section{Competing interests}


The authors declare that they have no competing interests.

\section{References}

1. Kubo T. Estimate of the number of night shift workers in Japan. J UOEH. 2014;36:273-6 (in Japanese).

2. The Japan Institute for Labour Policy and Training, https://www.jil.go.jil.go.jp/Kokunai/topics/mm/20181114.html. Accessed 28 Oct 2019 (in Japanese).

3. Akerstedt T. Sleepiness as a consequence of shift work. Sleep. 1988;11:17-34.

4. Scott LD, Hwang WT, Rogers AE, Nysse T, Dean GE, Dinges DF. The relationship between nurse work schedules, sleep duration, and drowsy driving. Sleep. 2007;30:1801-7.

5. Folkard S, Lombardi DA. Modeling the impact of the components of long work hours on injuries and "accidents". Am J Ind Med. 2006;49:953-63.

6. Salminen S. Long working hours and shift work as risk factors for occupational injury. Ergon Open J. 2016;9:15-26.

7. Tucker P; Smith L, Macdonald I, Folkard S. Shift length as a determinant of retrospective on-shift alertness. Scand J Work Environ Health. 1998;24:49-54.

8. Novak RD, Auvil-Novak SE. Focus group evaluation of night nurse shiftwork, difficulties and coping strategies. Chronobiol Int. 1996;13:457-63.

9. Smith A, Miles C. Acute effects of meals, noise and nightwork. Br J Psychol. 1986;77:377-87.

10. Gupta CC, Centofanti S, Dorrian J, Coates AM, Stepien JM, Kennaway DJ, et al. Subjective hunger, gastric upset, and sleepiness in response to altered meal timing during simulated shiftwork. Nutrients. 2019;11:1352.

11. Ishihara K, Miyashita A, Inugami M, Fukuda K, Yamazaki K, Miyata Y. The results of investigation by the Japanese version of Morningness-Eveningness Questionnaire. Shinrigaku Kenkyu. 1986;57:87-91 (in Japanese).

12. Baker FC, Driver HS. Circadian rhythms, sleep, and the menstrual cycle. Sleep Med. 2007;8:613-22.

13. Solomon SJ, Kurzer MS, Calloway DH. Menstrual cycle and basal metabolic rate in women. Am J Clin Nutr. 1982;36:611-6.

14. Faul F, Erdfelder E, Lang AG, Buchner A. G*Power 3: a flexible statistical power analysis program for the social, behavioral, and biomedical sciences. Behav Res Methods. 2007;39:175-91.

15. Mchill AW, Wright. KP Jr. Role of sleep and circadian disruption on energy expenditure and in metabolic predisposition to human obesity and metabolic disease. Obes Rev. 2017;18 Suppl 1:15-24.

16. Kawashima Y, Kakitsuba N. Optimal thermal conditions during night sleep in summer. J Human and Living Environment. 2004;11:17-23. (in Japanese) 
17. Härmä M, Waterhouse J, Minors D, Knanth P. Effect of masking on circadian adjustment and interindividual differences on a rapidly rotating shift schedule. Scand J Environ Health. 1994;20:55-

18. Romeijn N, Raymann RJ, Most E, Te Lindetr B, Van Der Meijden WP, Fronczek, R, et al. Sleep, vigilance, and thermosensitivity. Pflugers Archiv. 2012;463:169-76.

19. Purnell MT, Feyer AM, Herbison GP. The impact of a nap opportunity during the night shift on the performance and alertness of 12-h shift workers. J Sleep Res. 2002;11:219-27.

20. Monk TH. A visual analog scale technique to measure global vigor and affect. Psychiatry Res. 1989;27:89-99.

21. Sugimoto K, Kanai A, Shoji N. The effectiveness of the Uchida-Kreapelin test for psychological stress an analysis of plasma and salivary stress substances. Biopsychosoc Med. 2009, 4₫ doi: 10.1186/1751-0759-3-5.

22. Waggoner $L$, Grant $D$, Van Dogen $H$, et al. A combined field and laboratory design for assessing the impact of night shift work on police offer operational performance. Sleep, 2012;35:1575-7.

23. Roach GD, Dawson D, Lamond N. Can a shorter psychomotor vigilance task be used as a reasonable substitute for the ten-minute psychomotor vigilance task? Chronobiol Int. 2006;23:1379-87.

24. Wehrens SM, Hampton SM, Skene DJ. Heart rate variability and endothelial function after sleep deprivation and recovery sleep among male shift and non-shift workers. Scand J Work Environ Health. 2012;38:171-81.

25. Kamath MV, Fallen EL. Power spectral analysis of heart rate variability: a noninvasive signature of cardiac autonomic function. Crit Rev Biomed Eng. 1993;21:245-

26. Pagani M, Montano N, Porta A, Malliani A, Abboud FM, Birkrr C, et al. Relationship between spectral components of cardiovascular variabilities and direct measures of muscle sympathetic nerve activity in humans. Circulation. 1997;95:1441-

27. Zhang $C, Y u X$. Estimating mental fatigue based on electroencephalogram and heart rate variability. Pol J Med Phys Eng. 2010;16:67-84.

28. Van Dongen HP, Maislin G, Dinges DF. Dealing with inter-individual differences in the temporal dynamics of fatigue and performance: importance and techniques. Aviat Space Environ Med. 2004;75:147-54.

29. Akerstedt T. Shift work and disturbed sleep/wakefulness. Occup Med. 2003;53:89-94.

30. Oriyama S, Miyakoshi Y. The effects of nighttime napping on sleep, sleep inertia, and performance during simulated $16 \mathrm{~h}$ night work: a pilot study. J Occup Health. 2018;60:172-81.

31. Gupta CC, Dorrian J, Grant CL, Pajcin M, Coates AM, Kennaway DJ, et al. It's not just what you eat but when: the impact of eating a meal during simulated shift work on driving performance. Chronobiol Int. 2017;34:66-77.

32. Hallvig D, Anund A, Fors C, Kecklund G, Karlsson JG, Wahde M, et al. Sleepy driving on the real road and in the simulator-A comparison. Accid Anal Prev. 2013;50:44-50. 
33. Hirano Y, Obeta T, Takahashi H, Tachibana A, Kuroiwa D, Takahashi T, et al. Effects of chewing on cognitive processing speed. Brain Cogn. 2013;81:376-81. doi: 10.1016/j.bandc.2012.12.002.

34. Graw P, Kräuchi K, Knoblauch V, Wirz-Justice A, Cajochen C. Circadian and wake-dependent modulation of fastest and slowest reaction times during the psychomotor vigilance task. Physiol Behav. 2004;80:695-701.

35. Van Dongen HP, Maislin G, Mulligton JM, Dinges DF. The cumulative cost of additional wakefulness: dose-response effects on neurobehavioral functions and sleep physiology from chronic sleep restriction and total sleep deprivation. Sleep. 2003;26:117-26.

36. Grant CL, Coates AM, Dorrian J, Kennaway DJ, Wittert GA, Heilbronn LK, et al. Timing of food intake during simulated night shift impacts glucose metabolism: A controlled study. Chronobiol Int. 2017;34:1003-13.

37. Leung GKW, Huggins CE, Bonham MP. Effect of meal timing on postprandial glucose responses to low glycemic index meal: a crossover trial in healthy volunteers. Clin Nutr. 2019;38:465-71.

38. Mchill AW, Wright KP Jr. Role of sleep and circadian disruption on energy expenditure and in metabolic predisposition to human obesity and metabolic disease. Obes Rev. 2017;18 (Suppl 1): 1524

39. Kubo T, Fukuda S, Hirata K, Shimada K, Komukai K, et al. Comparison of coronary microcirculation in female nurses after day time versus night time shift. Am J Cardiol. 2011;108:1665-8.

40. Tepas D. Do eating and drinking habits interact with work schedule variables? Work \& Stress. 1990;4:203-11.

41. Van Cauter E, Polonsky KS, Scheen AJ. Roles of circadian rhythmicity and sleep in human glucose regulation. Endocr Rev. 1997;18:716-38.

42. Dye L, Lluch A, Blundell JE. Macronutrients and mental performance. Nutrition. 2000;16:1021-34.

43. Reyner LA, Wells SJ, Mortlock V, Horne JA. ‘Post-lunch' sleepiness during prolonged, monotonous driving-effects of meal size. Physiol Behav. 2012;105:1088-91.

44. Wells AS, Read NW, Idzikowski C, Jones J. Effects of meals on objective and subjective measures of daytime sleepiness. J Appl Physiol. 1998;84:507-15.

45. Smith AP, Kendrick AM, Maben AL. Effects of breakfast and caffeine on performance and mood in the late morning and after lunch. Neuropsychobiology. 1992;26:198-204.

46. Van Baak MA. Meal-induced activation of the sympathetic nervous system and its cardiovascular and thermogenic effects in man. Physiol Behay. 2008;94:178-86.

47. Collins SM, Karasek RA, Costas K. Job strain and autonomic indices of cardiovascular disease risk. Am J Ind Med. 2005;48:182-93.

48. Pejovic S, Vgontzas AN, Basta M, Tsaoussoglou M, Zoumakis E, Vgontzas A, et al. Leptin and hunger levels in young healthy adults after one night of sleep loss. J Sleep Res 2010;19:552-8.

49. Ohashi Y, Tachi N, Niwa S, Sakuragi Y, Kouyama U. Investigation of factors influencing fatigue in hospital nurses. J Jpn Soc Nurs Health Care. 2010;12:20-9 (in Japanese with English abstract). 
50. Saqah Zadeh R, Shepley M, Sadatsafavi H, Owora AH, Krieger AC. Alert workplace from healthcare workers' perspective: Behavioral and environment strategies to improve vigilance and alertness in healthcare settings. HERD. 2018;11:72-88.

51. Oriyama S, Miyakoshi Y, Rahman MM. The effects of a 120-minute nap on sleepiness, fatigue, and performance during 16-hour night shifts: a pilot study. J Occup Health. 2019;61: 368-77.

\section{Figures}

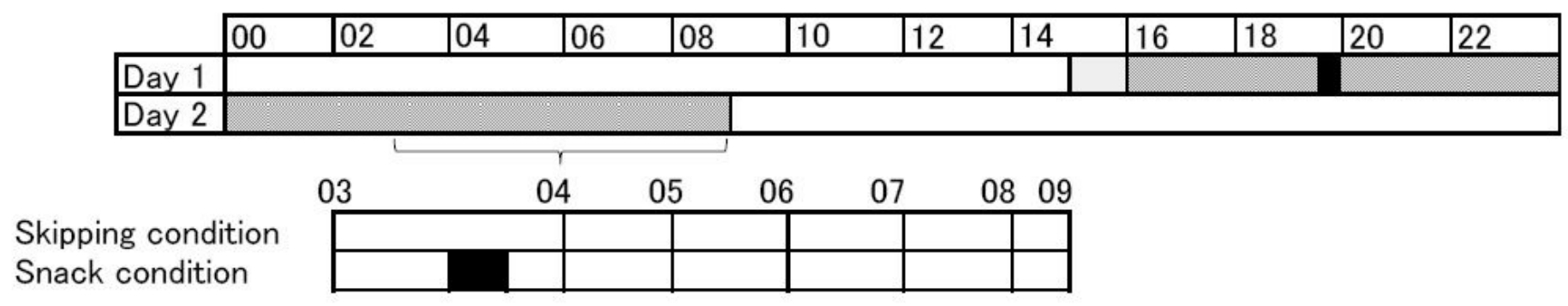

\section{Figure 1}

A flowchart of this study 

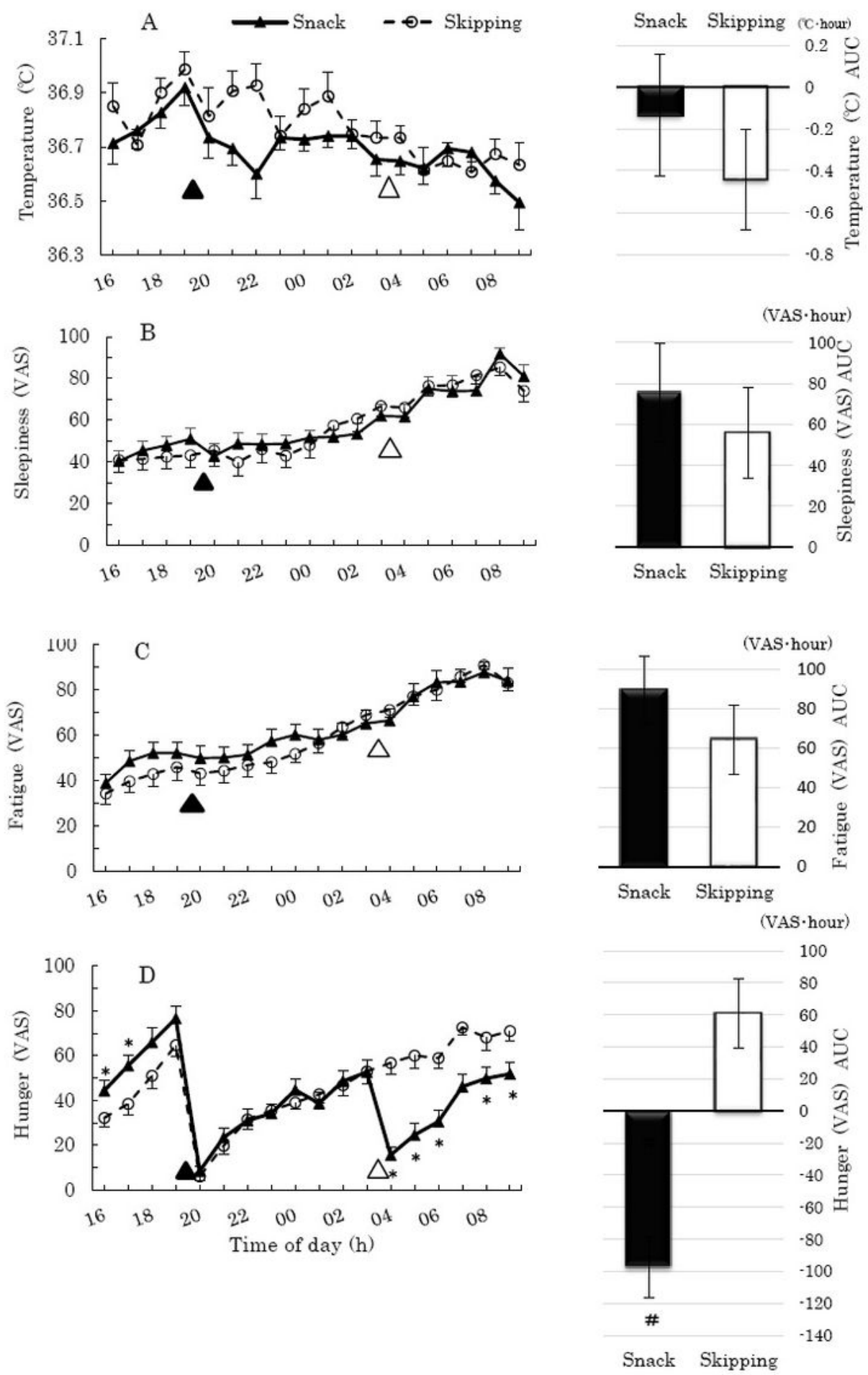

Figure 2

Outcomes regarding the participants' physiological parameters, cognitive performance, and autonomic nervous system activity from 16:00 to 09:00 

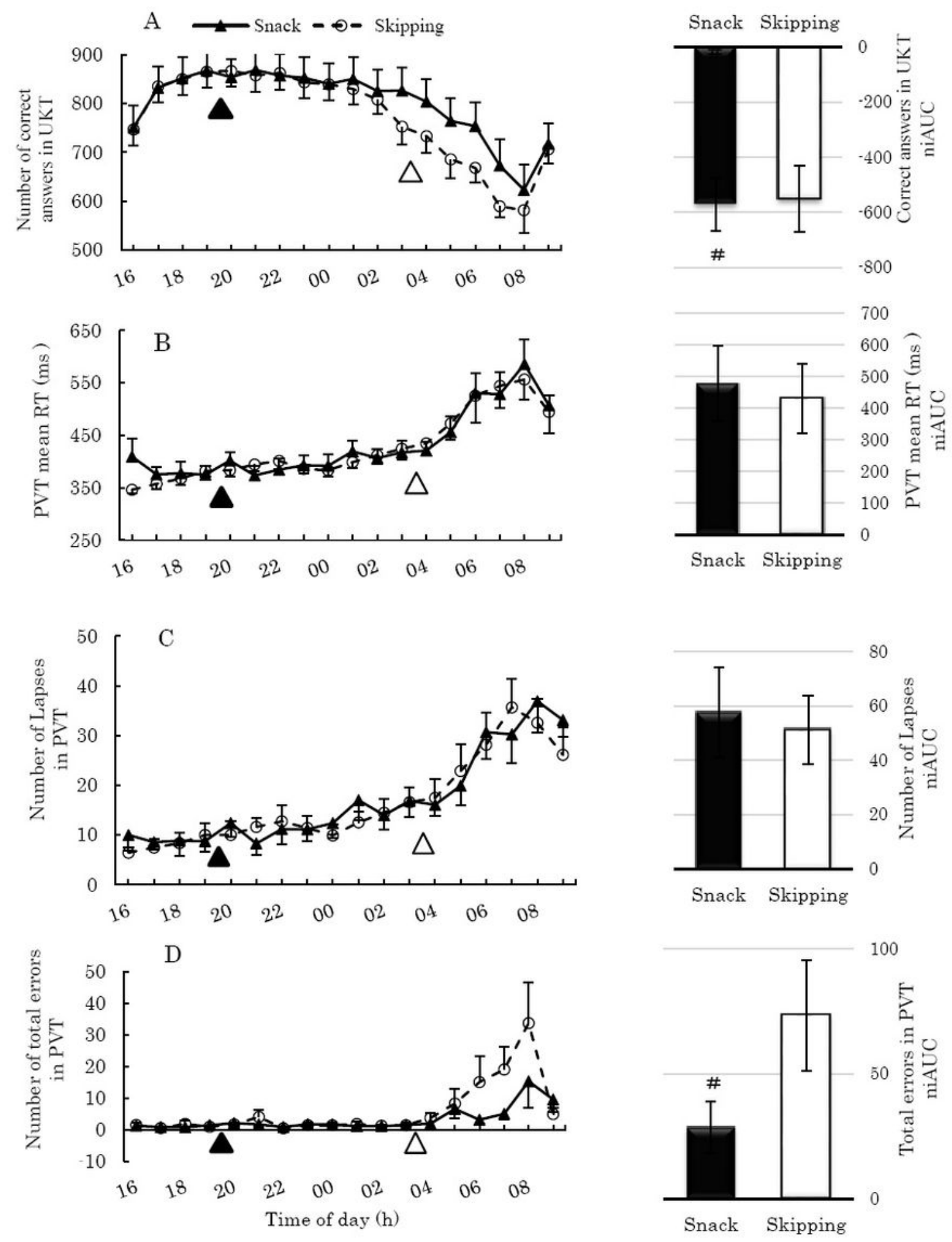

Figure 3

Results regarding the mean RT, lapse, and total errors on the PVT from 03:00 to 09:00 

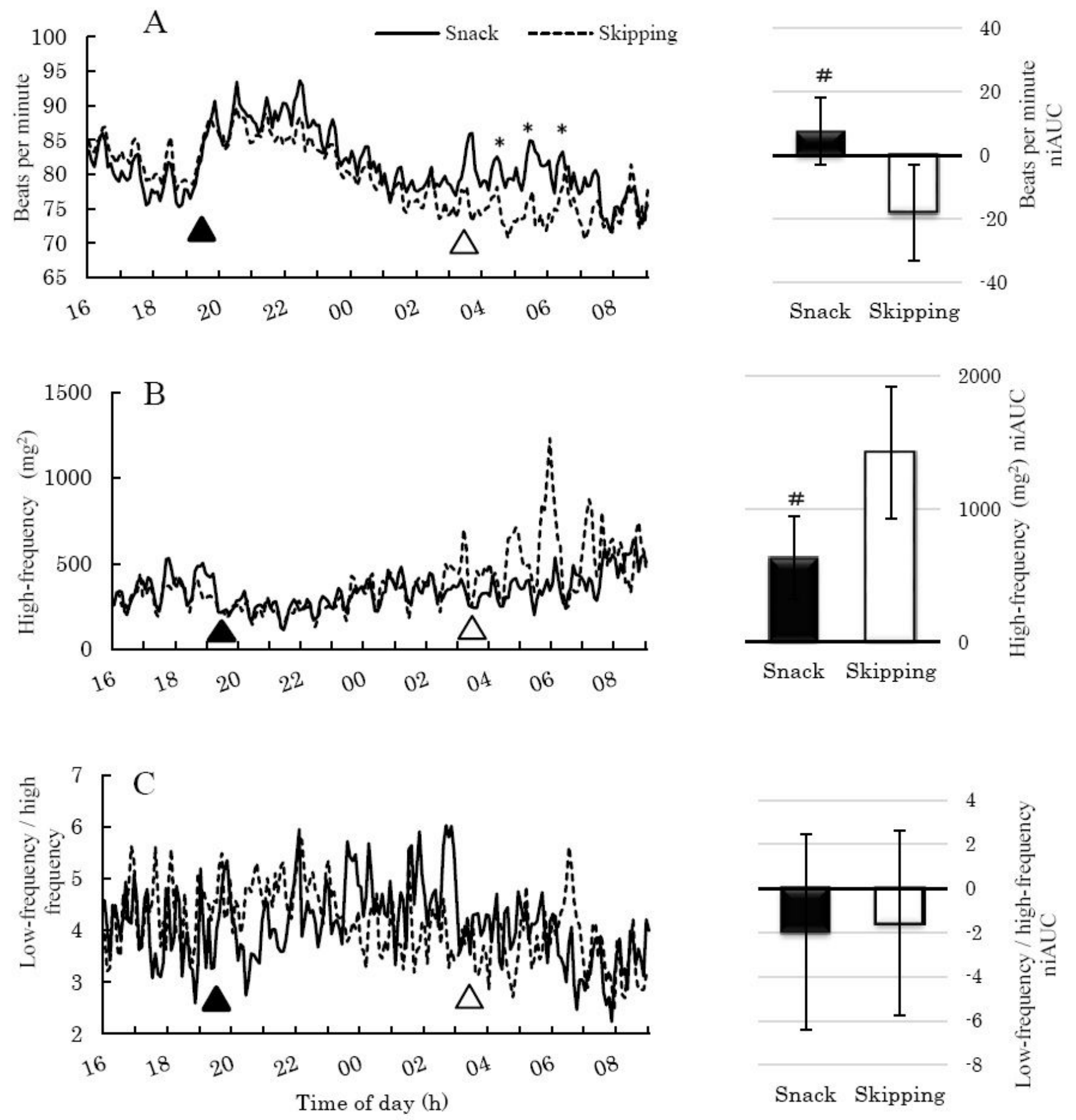

Figure 4

Electrocardiogram data could not be obtained from one participant during the experiment 